'workers at particular risk' and of health surveillance of exposed workers.

Shengli Niu ${ }^{1}$, Leena Korpinen ${ }^{2}$, Carlo Grandi ${ }^{3}$, Fabriziomaria Gobba ${ }^{4}$

${ }^{1}$ International Labour Office, Geneva, Switzerland

${ }^{2}$ Clinical Physiology and Neurophysiology Unit, The North Karelia Central Hospital, Joensuu, Finland

${ }^{3}$ INAIL - Department of Occupational and Environmental Medicine, Epidemiology and Hygiene, Monte Porzio Catone (ROMA), Italy

${ }^{4}$ University of Modena and Reggio Emilia, Modena, Italy

\section{9a ILO ACTIVITIES FOR THE PREVENTION OF THE RISK RELATED TO OCCUPATIONAL EXPOSURE TO EMF IN WORKERS}

S Niu. International Labour Office, Geneva, Switzerland

\subsection{6/oemed-2018-ICOHabstracts. 1202}

Introduction Hazards of non-ionising electromagnetic radiation (EMF) and risks from occupational exposures to them have been an increased concern to the ILO in particular over the last three decades. The health implications of over exposure to EMF are well documented in the scientific literature, such as injuries caused by leakage from microwave ovens or radiofrequency, and in some countries acute health effects of EMF can be considered occupational injuries. On the other hand there is only very limited scientific evidence presently on the diseases possibly caused by EMF exposure in workers. The ILO uses various means of action to give governments and employers' and workers' organisations the necessary help in drawing up and implementing programmes for the control of workplace risk factors, including EMF, as international standards in the form of legal instruments, codes of practice, practical manuals, training materials and education and training and technical cooperation. The ILO has collaborated with the ICNIRP and the WHO in the development of several practical guides and reports, including: Occupational Hazards from Non-Ionising Electromagnetic Radiation (ILO OSH Series No. 53), Protection of Workers From Power Frequency Electric and Magnetic Fields (ILO OSH Series No. 69), Visual Display Units: Radiation Protection Guidance (ILO OSH Series No. 70), and Safety in the Use of Radiofrequency Dielectric Heaters and Sealers (ILO OSH Series No. 71). These tools provide guidance on workplace safety and health measures and procedures that will lead to higher standards of safety for all personnel engaged in the operation which gives rise to occupational exposure to non-ionising radiation. The ILO also collects information on good workplace practice on protection of workers against non-ionising radiation and on the identifications and recognition of diseases as occupational caused by non-ionising radiation and provides support for the applications of the relevant ILO instruments in member States.

\section{9b EU DIRECTIVE 2013/35/EU ON OCCUPATIONAL EXPOSURE TO ELECTROMAGNETIC FIELDS}

${ }^{1} \mathrm{~L}$ Korpinen, ${ }^{2} \mathrm{R}$ Pääkkönen. ${ }^{1}$ Clinical Physiology and Neurophysiology Unit, The North Karelia Central Hospital, Joensuu, Finland; ${ }^{2}$ TMI Rauno Pääkkönen, Tampere, Finland
Introduction Directive 2013/35/EU of the European Parliament and of the Council on the minimum health and safety requirements regarding the exposure of workers to the risks arising from physical agents (electromagnetic fields (EMFs) was published in 2013. The directive gives minimum requirements for the protection of workers from risks to health and safety arising from exposure to electromagnetic fields $(0 \mathrm{~Hz}$ to $300 \mathrm{GHz}$ ). The aim of this paper is to describe the directive and how it has been implemented into practice.

Methods New guidelines have been written by different countries of the EU. Measurements and evaluations of EMFs have been conducted. High field values have been searched, and risk analyses for EMF exposure have been performed.

Results For example, the following topics have been proposed in new guidelines: offices, nuclear magnetic resonance (NMR) spectrometer, electrolysis, medical devices, engineering workshops, automotive industry, welding, metallurgical manufacturing, radiofrequency (RF) plasma devices, rooftop antennas, walkie-talkies, and airports. Evaluation criteria for the current is $100 \mathrm{~A}$, and for the voltages, $100 \mathrm{kV}$. There are also evaluation principles for active implanted devices, and there are guidelines on distance attenuation principles of EMFs.

Discussion There has been progress in implementing the directive. However, small and medium size companies have often limited resources to put toward evaluation and risk analysis. Protection against these fields can be technical, increasing distance, working processes, worker guides, or in some cases, personal protection devices. Health examination can also give in some cases information on excessive exposure (e.g., microwaves). Documentation of the actions is probably lacking to some extent in risk analysis files.

\section{C THE PROBLEM OF THE WORKERS AT PARTICULAR RISK}

C Grandi. INAIL - Dept. of Occupational and Environmental Medicine, Epidemiology and Hygiene, Monte Porzio Catone (RM), Italy

\subsection{6/oemed-2018-ICOHabstracts. 1204}

The directive 2013/35/EU on minimum health and safety requirements regarding the exposure of workers to risks arising from electromagnetic fields (EMF) states that the risk assessment and the implementation of preventive and protective measures have to take into account the workers at particular risk. Apart workers implanted with active or passive medical devices or wearing medical devices and pregnant women, the directive does not explicitly recognise other categories of workers at potential higher risk due to EMF exposure. Workers with active implanted medical devices (pacemakers in particular) are addressed by ad hoc technical standards (EN 50527-1:2010-04 and EN 50527-2-1:201105). In EU, active implantable and wearable medical devices must comply with requirements specified in technical standards, usually followed by the manufacturers. However, despite the growing diffusion of the active implantable or wearable medical devices and their diversification in terms of types and medical indications, other technical standards specifically devoted to workers carrying these devices and exposed to EMF are not yet available. For passive implanted medical devices, the risk assessment has to be performed mainly based on the available scientific literature. The case of pregnant women deserves special considerations while biological and pathological conditions making the worker at potential higher 